\title{
Susceptibility of stem infected sweet corn hybrids to common smut disease
}

\author{
${ }^{1}$ Dóra Frommer $-{ }^{2}$ Szilvia Veres $-{ }^{1}$ László Radócz \\ University of Debrecen, Faculty of Agricultural and Food Sciences and Environmental Management, \\ ${ }^{1}$ Institue of Plant Protection, Debrecen \\ ${ }^{2}$ Institute of Crop Sciences, Debrecen \\ dorafrommer@gmail.com
}

\section{SUMMARY}

The common smut of maize (corn smut, Ustilago maydis /DC/ Corda) can cause large economic losses in susceptible sweet corn hybrids as well. The protection against this pathogen is fundamentally based on prevention. Many methods to control corn smut have been recommended or evaluated, including crop rotation, sanitation, seed treatments, modification of fertility, and biological control. In spite of these frequently mentioned control strategies, the host resistance seems to be the only effective method to manage common smut in those areas where Ustilago maydis is prevalent.

Keywords: Ustilago maydis, susceptibility, stem infection

\section{INTRODUCTION}

Ustilago maydis, the causal agent of corn smut disease, is a biotrophic basidiomycete parasite on maize (Zea mays) and its natural ancestor teosinte species. It can induce the formation of tumors on all aerial organs (Banuett 1995) resulting stunted growth and yield losses (Martinez-Espinoza et al. 2002). Tumors are found most frequently on ears, tassels, stalks, nodal shoots, and mid-ribs of leaves. Smut galls consist of fungal and host tissues. Young galls are white, firm and covered with a semiglossy periderm. As galls begin to mature, interior tissue becomes semifleshy and streaks of black tissues occur as teliospores begin to form. During further maturation galls become a mass of black powdery teliospores and the periderm ruptures releasing the spores. Yield of infected plants can be reduced by 40 $100 \%$ (Shurtleff 1980). Losses vary with growing year, location, and cultivar. Ear development on infected plants may be impaired depending on the number, size and location of smut galls (Bojanowski 1969). Thus large galls particularly those above the ear, can cause barrenness of corn plants, multiple galls per plant also often reduce yield by $100 \%$ (Christensen 1963). Ustilago shows a dimorphic lifestyle (Kahmann and Kamper 2004), while haploid sporidia are not infectious and grow saprophytically in a yeast-like manner, filamentous growth is initiated upon mating of two compatible sporidia on the plant surface. Filamentous hyphae form appresoria and directly penetrate host cells. Immediately upon host cells and concomitant with tumor development the fungal proliferation is initiated. About four days after penetration, the formation of hypertrophic host cells and tumor development are induced, while the fungal hyphae start proliferating in the apoplastic spaces that develop as a consequence of cell wall degradation and induced host cells enlargement (Doehlemann et al. 2008).

Corn growers worldwide, particularly in dry or cold growing areas have experienced presence of common smut in their fields. The protection against this pathogen is basically based on prevention. Many methods of controlling corn smut have been recommended such as well-balanced soil fertility, possibly based on soil tests. Excessive nitrogen available in soil, accompanied sometimes by low phosphorus level, increases the chance for smut infestation. Very dry weather conditions tend to aggravate this further. Furthermore it is important to avoid mechanical injuries to plants. Implements could cause small cuts and wounds to the leaves, stalks, which then provide entry points for the fungus. The protection against insects is also essential. This can achieve in the early stage of crop development by insecticide seed treatment. Besides of these a frequently mentioned control alternative the host resistance which provides the most advantageous solution against the pathogen.

The variation in susceptibility of maize (field corn) hybrids to Ustilago maydis is very high. The mechanism of resistance to smut is not well understood, but various components of resistance have been postulated to be associated with physiological age and morphological characteristics of the maize variety (Christensen 1963). Sweet corn varieties are slightly more susceptible than other maize varieties because of low resistance in the germplasm from which modern sweet corn hybrids are derived (du Toit and Pataky 1999). Resistance to smut in field corn varieties has been increased by eliminating lines with susceptibility to Ustilago maydis from breeding programs. Current breeding programs rely on natural infection of smut in field plots and elimination of the most susceptible breeding lines in order to maintain smut resistance. Numerous reports are available on the influence of various inoculation techniques, host developmental stage and environmental conditions in pathogenesis (Kealey and Kosikowski 1981, Pataky 1991). Attempts have been made for inoculation corn methods that include: spraying, sandblasting, dusting, painting, dipping, injection and vacuum infiltrating spores on to plant parts, including stalks, tassel, bud, 
ears and roots with both teliospores and sporidia (Thakur et al. 1989, Zimmerman and Pataky 1992). Galls were induced in high frequency when asporidial suspension was injected between the leaf sheath and stalk at $6^{\text {th }}, 7^{\text {th }}$ and $8^{\text {th }}$ nodes below the top of plant $0-8$ days before tassel emergence (Thakur et al. 1989). The cob injection and tip injection methods consistently resulted in the highest incidence $97 \%$ and the highest severity of disease (Pope and McCarter 1992).

\section{MATERIALS AND METHODS}

The susceptibility of five different sweet corn (Zea mays L.) hybrids, viz. 'Box-R', 'Jumbo', 'Noa', 'Prelude' and 'Desszert 73' was investigated after corn smut (Ustilago maydis) artificial inoculation. The infection process took place after inoculation. The treatments in a randomized small plot field experiment were made in three repetitions at the growth stage of 6-7 leaves at BBCH 31 (Hanway 1963). The stem was inoculated at the second nodal height. Teliospores were collected and isolated from various types of infected maize smut. Fungus was grown in PSZA liquid and on solid media (Holiday 1961). Monosporidial colonies required to the inoculation were gained through dilution. Compatibility was specified based on the formation of aerial mycelium. The inoculation technique was injection. The inocula were provided by 1:1 mixture of the liquid culture grown for 24 hours of the two compatible monosporidial strains. The injection was performed using a special inoculation device. All the plants were injected with $2 \mathrm{ml}$ of sporidial suspension to induce a high incidence of common smut. The sporidium concentration of the suspension was $2000 \mathrm{ml}^{-1}$. The susceptibility of plants was evaluated 20 days after inoculation. Severity of common smut symptoms was evaluated using a rating scale (Table 1) based on the percentage of plants which have galls or typical symptoms of Ustilago maydis.

Infection rates for maize common smut

Table 1

1: no symptom

2: $1-3 \%$ of the plants are infected

(1 leaf has symptom)

3: $4-10 \%$ of the plants are infected

(2 leaves have symptoms)

4: $11-25 \%$ of the planta are infected

(3 leaves have symptoms)

5: $25-50 \%$ of the planta are infected

(4 leaves have symptoms)

6: $51-75 \%$ of the plants are infected

(4 leaves and tassel have symptoms)

7: 76-100\% of the planta are infected

(5 leaves and tassel have symptoms)

\section{RESULTS AND DISCUSSION}

Every hybrid developed typical symptoms of the diseases. The disease systematically spread in the plants after stem inoculation. The most characteristic symptom of the infected plants was the younger leaves turning into yellow colour. Furthermore there were observable slower and stunted growth of shoots, dwarfism, deformity and slanting. A distinctive symptom of Ustilago maydis infection was the formation of plant tumors. Among the susceptible hybrids the tassel also transformed into smut gall (Figure 1).

\section{Figure 1: Smut gall formation after stem inoculation}

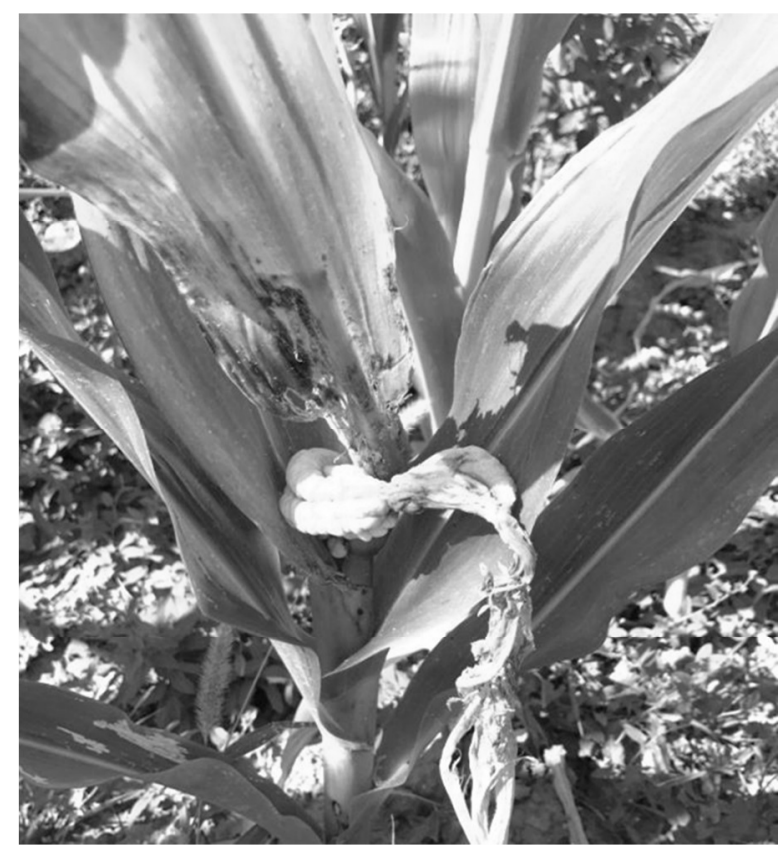

The most remarkable symptom induced in maize infected by Ustilago maydis is hypertrophy of plant cells, which is observable macroscopically in the form of tumors. Within these tumors a massive proliferation of fungal dikaryotic sporogenous hyphae takes place that later on differentiate into diploid teliospores (Kruger et al. 2000). As the number of mature teliospores increases, tumors becaming dark. These processes are not synchronized in different tumors, and even in different parts of the same tumor teliospore at different stages of development are encountered (Banuett and Herskowitz 1996). Even silk are infected, tumors develop in the form of enlarged, hollow ovaries with galled ovary walls (Snetselaar et. al. 2001).

On the base of visual evaluation each sweet corn hybrids resulted in different rate infections after stem inoculation by Ustilago maydis. None of the hybrids showed complete resistance to the disease, however different susceptibility could be experienced among sweet corn hybrids (Figure 2). 
Figure 2: Susceptibility of sweet corn hybrids after stem inoculation by common smut

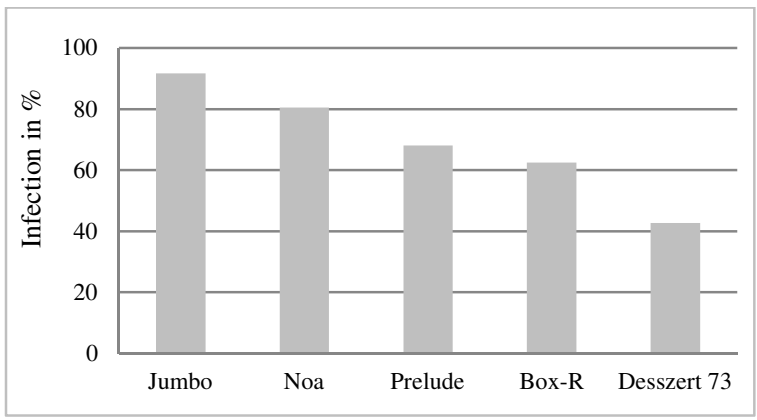

The smut incidence was very high in case of 'Jumbo' hybrid, the infection rate was $92 \%$ in average. Regarding 'Jumbo' hybrid serious symptoms such as dwarfism, tumors and deformity were experienced, moreover the whole tassel transformed to a large tumor. Concerning 'Desszert 73' hybrid the infection rate was the lowest, $43 \%$ in average. In the case of 'Noa' hybrid $81 \%$ infection rate was experienced. Regarding 'Prelude' hybrid the infection rate was $68 \%$ and concerning 'Box-R' hybrid the infection rate was $63 \%$ in average. The measured infection between the most sensitive 'Jumbo' hybrid and the less sensitive 'Desszert 73' hybrid the difference was $49 \%$.

\section{CONCLUSIONS}

The experiments proved that susceptibility to corn smut may vary among the tested hybrids. Five different sweet corn hybrids were studied after Ustilago maydis artificial inoculation. All hybrids developed typical symptoms of the diseases, none of them showed resistance to common smut. However, differences were noticed in susceptibility among the examined hybrids. The most characteristic symptom of the infected plants was that leaves were turning into yellow colour. Furthermore slow and stunted growth of shoots, dwarfism, deformity and slanting were experienced. A distinctive symptom of the Ustilago maydis infection was the formation of plant tumors involving cell proliferation.

The differences regarding the susceptibility of the hybrids were determined based on the infection rate. The sweet corn 'Jumbo' hybrid was the most susceptible, and the less susceptible was 'Desszert 73' hybrid. It seems that the examined sweet corn hybrids are more or less sensitive to common smut disease caused by Ustilago maydis.

\section{REFERENCES}

Banuett, F. (1995): Genetics of Ustilago maydis, a fungal pathogen that induces tumors in maize. Annu. Rev. Genet. 29: 179-208.

Banuett, F.-Herskowitz, I. (1996): Discrete developmental stages during teliospore formation in the corn smut fungus, Ustilago maydis. Development. 122: 2965-2967.

Bojanowski, J. (1969): Studies of inheritance of reaction to common smut in corn. Appl. Genet. 39: 32-42.

Christensen, J. J. (1963): Corn smut caused by Ustilago maydis. Am. Phytopathol. Soc. Monogr. 2: 35-41.

Doehlemann, G.-Wahl, R.-Horst, R. J.-Voll, L.-Usadel, B.-Poree F.-Stitt, M.-Pons-Kuehnemann, J.-Sonnenwald, U.-Kahmann, R. (2008): Reprogramming a maize plant: transcriptional and metabolic changes induced by the fungal biotroph Ustilago maydis. Plant J. 56: 181-195.

du Toit, L. J. - Pataky, J. K. (1999): Variation associated with silk channel inoculation for common smut of sweet corn. Plant Dis 83: 727-732.

Hanway, J. J. (1963): Growth stages of corn. Agronomy Journal. 55: 487-492

Holiday, R. (1961): The genetics of Ustilago maydis. Genet. Red. Camb. 2: 204-230.

Kahmann, R.-Kamper, J. (2004): Ustilago maydis: how its biology relates to pathogenetic development. New Phytol. 164: 31-42.

Kealey, K. S.-Kosikowski, F. Y. (1981): Corn smut as a food source - Perspectives on biology, composition and nutrition. CRC Crit. Rev. Food Sci. Nut. 15: 321-351.
Kruger, J.-Loubradou, G.-Wanner, G.-Regenfelder, E.Feldbrugge, M.-Kahmann, R. (2000): Activation of the cAMP pathways in Ustilago maydis reduces fungal poliferation and teliospore formation in plant tumors. Mol. Plant Microbe Interact. 13: 1034-1040.

Martinez-Espinoza, A. D.-Garcia-Pedrajas, M. D.-Gold, S. E. (2002): The Ustilaginales as plant pests and model systems. Fungal Genet. Biol. 35: 1-20.

Pataky, J. K. (1991): Production of cuitlacoche (Ustilago maydis (DC) Corda) on sweet corn. Hort. Science. 26: 1374-1377.

Pope, D. D.-McCarter, S. M. (1992): Smut incidence and severitiy after inoculating developing corn ears with Ustilago maydis using different methods. Phytopathology. 82: 500.

Shurtleff, M. C. (1980): Compendium of Corn Diseases. $2^{\text {nd }}$ edition. The Diseases Compendia Series. The American Phytopath. Society.

Snetselaar, K. M.-Carfioli, M. A.-Cordisco, K. M. (2001): Pollination can protect maize ovaries from infection by Ustilago maydis, the corn smut fungus. Can. J. Bot. 79: 13901399.

Thakur, R. P.-Leonard, K. J.-Pataky, J. K. (1989): Smut gall development in adult corn plants inoculated with Ustilago maydis. Plant Dis. 73: 921-925.

Zimmermn, S. A. - Pataky, J. K. (1992): Inoculation techniques to produce galls of common smut on ears of sweet corn. Phytopathology. 82: 995. 